\title{
Variability of leaf pubescence characteristics in transgenic tobacco lines with partial proline dehydrogenase gene suppression
}

\author{
S.M. IBRAGIMOVA* (D), M.A. GENAEV (D), A.V. KOCHETOV (D), and D.A.AFONNIKOV* \\ Federal Research Center Institute of Cytology and Genetics, and Kurchatov Genomic Center of the Institute of \\ Cytology and Genetics, Siberian Branch of Russian Academy of Sciences, Novosibirsk, 630090, Russian Federation
}

*Corresponding authors: E-mails: isola@bionet.nsc.ru, ada@bionet.nsc.ru

\begin{abstract}
Proline, an amino acid, plays an important role in plants, and it is involved in stress resistance and development. Earlier, to study the proline role in maintaining stress resistance in plants, we obtained genetically modified transgenic lines of tobacco (Nicotiana tabacum L.) with reduced activity of proline dehydrogenase ( $P D H$, the proline degradation gene) and increased content of proline. Transgenic tobacco plants demonstrated greater resistance to high concentrations of $\mathrm{NaCl}$, drought, low temperatures, and heavy metals vs. control plants. The visual assessment showed that the leaf pubescence in transgenic plants varied noticeably. Here we apply automated analysis of the tobacco leaf folds to estimate quantitative characteristics of pubescence in genetically modified tobacco plants and the control SR1 line under nonstress conditions. Our results showed differences in the number of trichomes and their length between transgenic and control plants. The trichome number significantly increased in transgenic plants (from 1.5 to 3 times). The largest differences in the trichome numbers were observed for trichomes with lengths from 0 to $380 \mu \mathrm{m}$. When assessing the trichome length, the opposite was observed. In all three transgenic lines, the trichome length was significantly lower than that of the control SR1 line. The data obtained indicate the effect of proline as an important metabolome component affecting the plant phenotype. Our results demonstrate perspectives of tobacco transgenic lines as promising genetic models for studying the proline role in plant morphogenesis.
\end{abstract}

Keywords: image analysis, leaf trichomes, Nicotiana tabacum, proline, proline dehydrogenase, transgenic lines.

Proline plays an important role in plant ontogenesis and stress response (Dar et al. 2016, Trovato et al. 2019). The proline content in plant cells increases manifold in response to an increase or decrease in temperature, to drought, soil salinity, nutrient deficiency, increased UV radiation, or exposure to heavy metals resulting in plant osmotic stress (Kuznetsov and Shevyakova 1999). An increase in cell proline leads to the modulation of cell pressure potential, thereby creating an osmotic balance, stabilizes cell membranes, protein and enzyme structures, preventing electrolyte leakage in the cell and oxidative stress. Thus, proline acts as a signalling molecule of stress response in plants (Hayat et al. 2012).

Changes in proline content are just one of many plant stress responses: physiological, morphological, and anatomical (Hameed et al. 2010, Ilyas et al. 2020). One of the characteristic morphological changes is associated with leaf pubescence. Leaf pubescence is formed by

$\overline{\text { Received }} 9$ June 2021, last revision 18 October 2021, accepted 10 November 2021.

Abbreviations: PDH - proline dehydrogenase.

Acknowledgements: This work was supported by funding from the Kurchatov Genome Center of the Federal Research Center of the Institute of Cytology and Genetics, Siberian Branch of the Russian Academy of Sciences, Agreement with the Ministry of Education and Science of the Russian Federation No. 075-15-2019-1662. Plant growing in the ICG Plant Growth Core Facility was supported by the budget project No. 0259-2021-0012. The authors express their appreciation to V.F. Fedotova for technical assistance in the work. Conflict of interest: The authors declare that they have no conflict of interest. 
Table 1. Quantitative characteristics of leaf pubescence (number of trichomes and their length) in tobacco transgenic lines (Pdh) and control line SR1 (wild type). Means \pm SEs, parameters that significantly differ in transgenic lines from the control are marked with * $(P<0.01)$, Var $\left(\mathrm{n}_{\mathrm{f}}\right)$, Var $\left(\mathrm{l}_{\mathrm{m}}\right)$ - variances of the $\mathrm{n}_{\mathrm{f}}$ and $\mathrm{l}_{\mathrm{m}}$ parameters, respectively; $r\left(\mathrm{n}_{\mathrm{f}}, 1_{\mathrm{m}}\right)$ - Pearson correlation coefficients between them.

\begin{tabular}{llcclrl}
\hline Lines & $\begin{array}{l}\text { Number of analyzed } \\
\text { images }\end{array}$ & $\begin{array}{l}\text { Number of trichomes } \\
\text { per image }\left(\mathrm{n}_{\mathrm{f}}\right)\end{array}$ & $\operatorname{Var}\left(\mathrm{n}_{\mathrm{f}}\right)$ & Trichome length, $1_{\mathrm{m}},[\mu \mathrm{m}]$ & $\operatorname{Var}\left(\mathrm{l}_{\mathrm{m}}\right)$ & $r\left(\mathrm{n}_{\mathrm{f}}, 1_{\mathrm{m}}\right)$ \\
\hline SR1 & 53 & $4.78 \pm 0.35$ & 1.67 & $308.39 \pm 19.82$ & 5271.98 & -0.06 \\
Pdh5 & 15 & $7.10 \pm 0.75^{*}$ & 2.14 & $250.36 \pm 22.32^{*}$ & 1857.82 & -0.44 \\
Pdh6 & 16 & $7.75 \pm 0.78^{*}$ & 2.26 & $229.74 \pm 12.50^{*}$ & 586.89 & -0.06 \\
Pdh8 & 24 & $13.19 \pm 0.72^{*}$ & 3.02 & $273.57 \pm 16.98^{*}$ & 690.47 & -0.13 \\
\hline
\end{tabular}
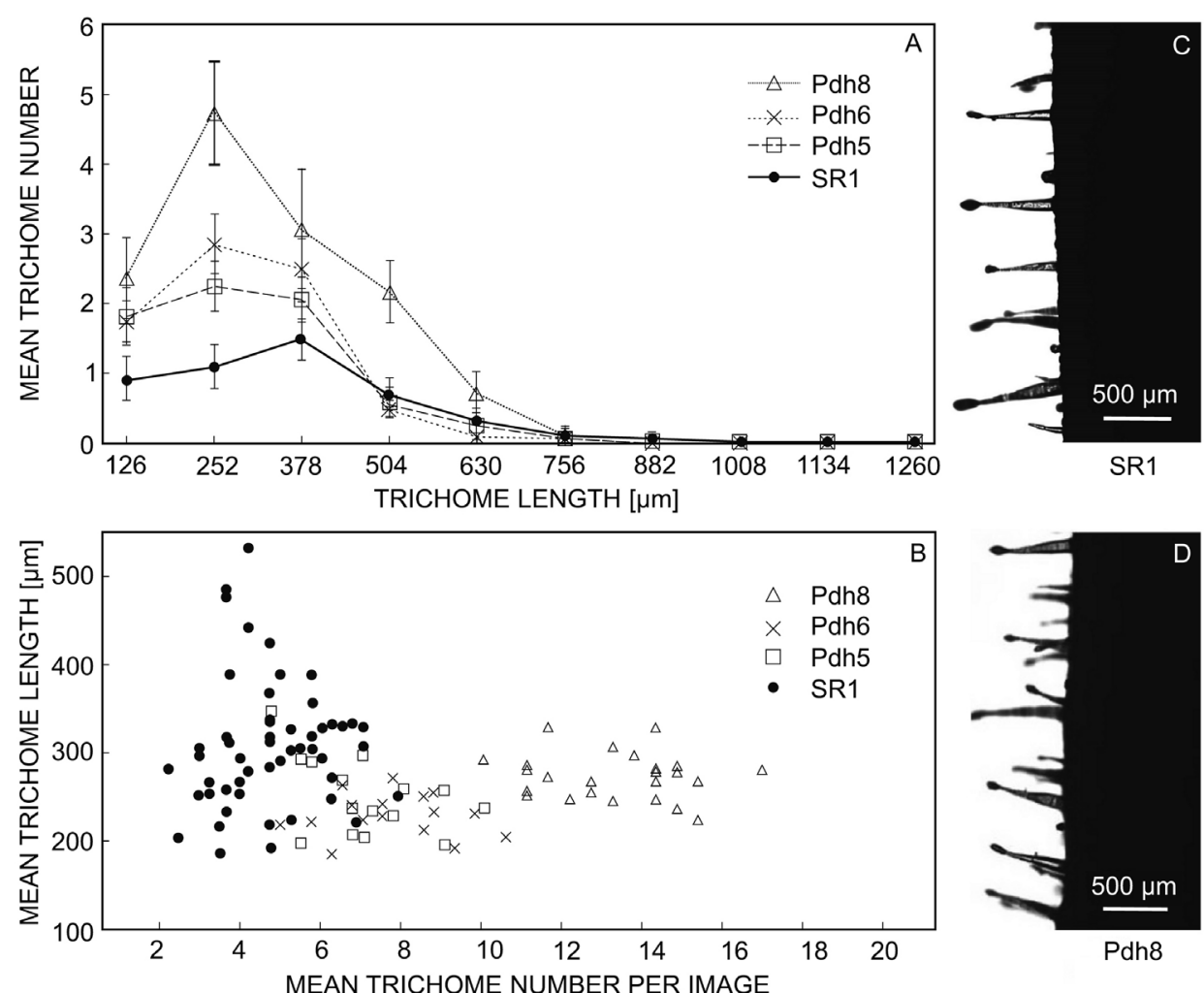

Fig. 1. Comparison of quantitative characteristics of leaf pubescence of the control (SR1) and three transgenic tobacco lines Pdh 5, Pdh 6, and Pdh 8. $A$ - Frequency polygons of the trichome length distribution in the image: the x-axis shows the trichome length intervals with a $126 \mu \mathrm{m}$ increment; the y-axis shows the trichome number in the length interval averaged over all the images; vertical lines show $95 \%$ confidence intervals for the mean value of the trichome number. $B$ - Scatterplot of tobacco leaf samples for four genotypes according to the mean trichome number per image (x-axis) and mean trichome length (y-axis). $C, D$ - The leaf fold image of the tobacco control SR1 line and the Pdh8 transgenic line used for data extraction.

trichomes and in most plant species functions as the first barrier of protection against abiotic and biotic stresses (Shvachko et al. 2020). Pubescence mechanically prevents the penetration of pests and their larvae, pathogenic and parasitic fungi to the plant mesophilic tissues preventing their damage. Trichomes can also accumulate heavy metals, thereby participating in their detoxification processes. In unfavourable environmental conditions, trichomes are involved in maintaining water balance and can protect underlying tissues from bright radiation increasing reflection and lowering the temperature (Hauser 2014).

Earlier, to study the role of proline in maintaining stress resistance in plants, we obtained model transgenic lines of tobacco (Pdh5, Pdh6, and Pdh8) with reduced activity of proline dehydrogenase $(\mathrm{PDH})$, the proline degradation gene, and increased content of proline in leaves in comparison with the control line, SR1 (Kochetov et al. 2004). It was shown that tobacco plants with reduced PDH activity exhibited elevated stress resistance to salinity, drought, low temperatures, and heavy metal salts (Kolodyazhnaya et al. 2006, Ibragimova et al. 2012, 2015). In addition to the manifestation of plant resistance to various stress types, preliminary analysis showed that the pubescence morphology in the transgenic lines and the control line were different. However, it was difficult to 
reliably assess the difference in pubescence by visual and tactile inspection.

Here, we assess the quantitative characteristics of leaf pubescence in tobacco transgenic lines with a reduced $P D H$ gene activity and increased proline content using high throughput plant phenotyping (Afonnikov et al. 2016). We estimate the trichome number per image and length using the automated leaf fold image analysis. The method was developed for wheat (Genaev et al. 2012) and adapted for tobacco.

Plants of the tobacco transgenic lines (Pdh5, Pdh6, Pdh8) and the control SR1 line (Kochetov et al. 2004) were grown in a greenhouse (Laboratory of Artificial Cultivation of Plants of the Institute of Cytology and Genetics of the SB RAS, Novosibirsk) in pots with clay pellets and regular watering with Knopp solution (a 16-h photoperiod, a temperature of $24-25{ }^{\circ} \mathrm{C}$, air humidity of $50-60 \%$, and irradiance of $400 \mu \mathrm{mol} \mathrm{m} \mathrm{m}^{-2} \mathrm{~s}^{-1}$ as additional light in the morning and evening. During the day, lighting was natural.). Five months old tobacco (Nicotiana tabacum L.) plants at the flowering stage were used for analysis. For the preparation of leaf folding, we took well-formed leaves from the middle layer, without signs of damage and deformation. Leaf samples for determining the pubescence characteristics were prepared according to the protocol given in the work of Doroshkov et al. (2016). From each analyzed leaf, at least three fragments of $10 \times 20 \mathrm{~mm}$ were cut out between the leaf radial veins, bent in half, so that the adaxial surface is presented to view. Folded fragments were placed on a glass slide and fixed with adhesive tape a few millimetres from the fold edge. The pubescence parameters were evaluated on leaf samples collected from 5 plants of each of the studied lines. The images were obtained by a Carl Zeiss (Jena, Germany) Axioskop 2 plus microscope equipped with AxioCam HRc camera at lens magnification X5 in the Multiple-Access Center of the Institute of Cytology and Genetics SB RAS.

The mean trichome number per image $\left(n_{f}\right)$ and the mean trichome length $\left(1_{\mathrm{m}}\right)$, as well as the estimate of the Pearson correlation coefficient for these two values in a plant sample $(r)$, were estimated for each genotype and shown in Table 1. It should be noted that we estimated characteristics of the total pool of trichomes without discriminating them into two types (Akers et al. 1978). It turned out that the mean values of the trichome number and their mean length for tobacco transgenic lines significantly $(P<0.01)$ differed from those in plants of the control SR1 genotype. For the Pdh5 genotype, the $P$-values for the differences in the $\mathrm{n}_{\mathrm{f}}$ and $\mathrm{l}_{\mathrm{m}}$ parameters were below $1.7 \times 10^{-5}$ and 0.0004 , respectively. For the Pdh6 genotype, the $P$-values for these parameters were below $3.61 \times 10^{-7}$ and $4.6 \times 10^{-9}$, respectively. For the Pdh 8 line, the $P$-values for these parameters were below $1.5 \times 10^{-21}$ and 0.002 , respectively. It turned out that the trichome number in all genetically modified plants increased in comparison with the control line (Table 1). So, for the Pdh5 and Pdh6 lines, the value was almost one and a half times more, and for the Pdh 8 line almost three times. For the average trichome length, on the contrary, this parameter for plants of all transgenic lines was lower than for control plants.
The greatest difference in length (1.3 times) was observed for Pdh6 line plants. Analysis of Pearson's correlation coefficients between the trichome number and their average length in tobacco leaves showed a weak negative correlation: the values of the correlation coefficients were close in modulus to zero and were negative both in the control SR1 line and in two transgenic lines (Pdh6 and Pdh8). For the Pdh5 line, the correlation coefficient $r\left(n_{\mathrm{f}}, 1_{\mathrm{m}}\right)$ was high in absolute value $(-0.44)$ but still did not significantly differ from $0(P<0.09)$. Thus, no statistical relationship was found between the trichome number and their mean length.

Table 1 also shows that in the series of Pdh5-Pdh6Pdh8 genotypes, there was an increase in the trichome number per image, but no trends were observed for the length.

The folded leaf image analysis allows obtaining the trichome length distribution for each genotype. Frequency polygons drawn for trichome lengths with a bin interval of $126 \mu \mathrm{m}$ (Fig. 1A) showed that the strongest differences between the trichome number in the SR1 line and transgenic lines were in the 0 to $380 \mu \mathrm{m}$ length intervals. Such differences were expressed both when comparing plants of transgenic lines and the control SR1 line, and when comparing transgenic lines with one another. With trichome length within $400-600 \mu \mathrm{m}$, the differences between the trichome numbers were significant for the SR 1 and Pdh8 lines; for Pdh5 and Pdh6 lines, differences were vanishing. The number of trichomes with lengths more than $700 \mu \mathrm{m}$ approached zero for all genotypes. Note that in the case of trichome length distribution for the transgenic lines, a peak was observed in the region of $252 \mu \mathrm{m}$, while for SR1, the peak was shifted to the region of greater length $(378 \mu \mathrm{m})$, and for intervals of greater length, the trichome number in this line was more than in Pdh5 and Pdh6. This was probably the reason for the greater value of the average trichome length for the SR1 line (Table 1).

A scatterplot for tobacco leaf samples $n_{f}$ and $l_{m}$ parameters is shown in Fig. $1 B$. Samples of the control SR1 line were located in the region of low values of the trichome number ( 2 to 8$)$. The range of the trichome number in the image for the Pdh5 and Pdh6 lines was almost the same $(4-11)$ which determined the similarity of the average values of this parameter for two plant groups (Table 1). The $\mathrm{n}_{\mathrm{f}}$ values for the Pdh8 line were in the range from 10 to 17 ; the distribution had negligible overlap with the $\mathrm{n}_{\mathrm{f}}$ distribution for other genotypes. The mean trichome lengths for the plants of the transgenic lines were close (for all genotypes, the range is within $200-300 \mu \mathrm{m}$ ), and samples of the SR1 genotype demonstrated greater variations within $180-530 \mu \mathrm{m}$ (a noticeable number of samples had an average trichome length more than 300). As result, a higher value of the trichome mean length and variance in SR1 plants was observed (Table 1). Thus, the results demonstrated that tobacco transgenic lines with an altered pattern of the $P D H$ gene expression and an increased proline content had a greater trichome number on the adaxial leaf surface and shorter trichomes in comparison with the control SR1 line. 
Leaf pubescence is a dynamic trait that undergoes significant changes under stress conditions. The trichome density on the leaf surface increases in response to biotic and abiotic stresses in many plant species (Karabourniotis et al. 2020). Using the method developed earlier for phenotyping wheat leaf pubescence, it was shown that a more severe environment (Doroshkov et al. 2011) or the drought impact on wheat (Pshenichnikova et al. 2019) lead to an increase in the trichome density on the leaf and a decrease in their length.

An increasing number of accumulated data indicates proline participation in plant ontogenesis in the low-stress environment (Trovato et al. 2019). It provides plant cells of vegetative tissues with energy, contributing to their faster growth and development (Mattioli et al. 2009). Low proline content leads to impaired cytokinesis and the formation of abnormal embryos, which has been shown in maize (Zadebagheri et al. 2014). It is known that proline plays an important role in cell division, participates in the gene regulation of the cell cycle, in particular, cyclins, and is also a structural component of the cell wall (Roda et al. 2003, Biancucci et al. 2015).

Under stress conditions, a change in the leaf pubescence of plants at a high proline content can make an additional contribution to the organism's resistance to stress. In this work, a similar effect is observed at the constantly high content of proline in tobacco transgenic lines in the absence of stress conditions. The increased proline in tobacco transgenic lines contributes to more intensive cytokinesis of epidermal cells and results in the increased formation of trichomes.

The revealed fact of increased leaf pubescence in transgenic lines with an increased free proline deserves attention. Tobacco is one of the economically important industrial crops. Virtually all tobacco organs are covered with glandular trichomes. They store and secrete terpenes, sugars, nicotine, fatty acid derivatives, and flavonoids (Huchelmann et al. 2017). The data obtained indicate the effect of proline as an important metabolome component on the plant phenotype, and tobacco transgenic lines are promising genetic models for studying the proline role in plant morphogenesis.

\section{References}

Afonnikov, D.A., Genaev, M.A., Doroshkov, A.V., Komyshev, E.G., Pshenichnikova, T.A.: Methods of high-throughput plant phenotyping for large-scale breeding and genetic experiments. - Russ. J. Genet. 52: 688-701, 2016.

Akers, C.P., Weybrew, J.A., Long, R.C.: Ultrastructure of glandular trichomes of leaves of Nicotiana tabacum L., cv. Xanthi. - Amer. J. Bot. 65: 282-292, 1978.

Biancucci, M., Mattioli, R., Moubayidin, L., Sabatini, S., Costantino, P., Trovato, M.: Proline affects the size of the root meristematic zone in Arabidopsis. - BMC Plant Biol. 15: 263, 2015.

Dar, M.I., Naikoo, M.I., Rehman, F., Naushin, F., Khan, F.A.: Proline accumulation in plants: roles in stress tolerance and plant development. - In: Iqbal, N., Nazar, R. A., Khan, N. (ed.): Osmolytes and Plants Acclimation to Changing Environment:
Emerging Omics Technologies. Pp. 155-156. Springer, New Delhi 2016.

Doroshkov, A.V., Genaev, M.A., Afonnikov, D.A.: A protocol for analysis of the quantitative characteristics of leaf pubescence in potato. - Vavilovskii Zh. Genet. Selekt. 20: 863-868, 2016.

Doroshkov, A.V., Pshenichnikova, T.A., Afonnikov, D.A: Morphological characterization and inheritance of leaf hairiness in wheat (Triticum aestivum L.) as analyzed by computer-aided phenotyping - Russ. J. Genet. 47: 739-743, 2011.

Genaev, M.A., Doroshkov, A.V., Pshenichnikova, T.A., Kolchanov, N.A., Afonnikov, D.A.: Extraction of quantitative characteristics describing wheat leaf pubescence with a novel image-processing technique. - Planta 236: 1943-1954, 2012.

Hameed, M., Ashraf, M., Ahmad, M.S.A., Naz, N.: Structural and functional adaptations in plants for salinity tolerance. - In: Ashraf, M., Ozturk, M., Ahmad, M.S.A. (ed.): Plant Adaptation and Phytoremediation. Pp. 151-170. Springer, Dordrecht 2010.

Hauser, M.T.: Molecular basis of natural variation and environmental control of trichome patterning. - Front Plant Sci. 3: 320, 2014.

Hayat, S., Hayat, Q., Alyemeni, M.N., Wani, A.S., Pichtel, J., Ahmad, A.: Role of proline under changing environments: a review. - Plant Signal Behav. 7: 1456-1466, 2012.

Huchelmann, A., Boutry, M., Hachez, C.: Plant glandular trichomes: natural cell factories of high biotechnological interest. - Plant Physiol. 175: 6-22, 2017.

Ibragimova, S.S., Kolodyazhnaya, Ya.S., Gerasimova, S.V., Kochetov, A.V.: Partial suppression of gene encoding proline dehydrogenase enhances plant tolerance to various abiotic stresses. - Russ. J. Plant Physiol. 59: 88-96, 2012.

Ibragimova, S.M., Trifonova, E.A., Filipenko, E.A., Shymny, V.K.: Evaluation of salt tolerance of transgenic tobacco plants bearing the P5CS1 gene of Arabidopsis thaliana. - Russ. J. Genet. 51: 1181-1188, 2015.

Ilyas, M., Nisar, M., Khan, N., Hazrat, A., Khan, A.H., Hayat, K., Fahad, S., Khan, A., Ullah, A.: Drought tolerance strategies in plants: a mechanistic approach. - J. Plant Growth Regul. 40: 926-944, 2020.

Karabourniotis, G., Liakopoulos, G., Nikolopoulos, D., Bresta, P.: Protective and defensive roles of non-glandular trichomes against multiple stresses: structure-function coordination. - J. Forest. Res. 31: 1-12, 2020.

Kolodyazhnaya, Y.S., Titov, S.E., Kochetov, A.V., Komarova, M.L., Romanova, A.V, Koval, V.S., Shumny, V.K.: Evaluation of salt tolerance in Nicotiana tabacum plants bearing an antisense suppressor of the proline dehydrogenase gene. Russ. J. Genet. 42: 212-214, 2006.

Kochetov, A.V., Titov, S.E., Kolodyazhnaya, Y.S., Komarova, M.L., Koval, V.S., Makarova, N.N., Il'yinskyi, Yu.Yu., Trifonova, E.A., Shumny, V.K.: Tobacco transformants bearing antisense suppressor of proline dehydrogenase gene, are characterized by higher proline content and cytoplasm osmotic pressure. - Russ. J. Genet. 40: 216-218, 2004.

Kuznetsov, V.V., Shevyakova, N.I.: Proline under stress: biological role, metabolism, and regulation. - Russ. J. Plant Physiol. 46: 274-289, 1999.

Mattioli, R., Costantino, P., Trovato, M.: Proline accumulation in plants: not only stress. - Plant Signal Behav. 4: 1016-1018, 2009.

Pshenichnikova, T.A., Doroshkov, A.V., Osipova, S.V., Permyakov, A.V., Permyakova, M.D., Efimov, V.M., Afonnikov, D.A.: Quantitative characteristics of pubescence in wheat (Triticum aestivum L.) are associated with photosynthetic parameters under conditions of normal and 
limited water supply. - Planta 249: 839-847, 2019.

Roda, A.L., Oldham, N.J., Svatos, A., Baldwin, I.T.: Allometric analysis of the induced flavonols on the leaf surface of wild tobacco (Nicotiana attenuata). - Phytochemistry 62: 527-536, 2003.

Shvachko, N.A., Semilet, T.V., Tikhonova, N.G.: Higher plants trichomes: homologous series in hereditary variability and molecular genetic mechanisms - Russ. J. Genet. 56: 13201332, 2020.
Trovato, M., Forlani, G., Signorelli, S., Funck, D.: Proline metabolism and its functions in development and stress tolerance. - In: Hossain, M., Kumar, V., Burritt, D., Fujita, M., Mäkelä, P. (ed.): Osmoprotectant-mediated Abiotic Stress Tolerance in Plants. Pp. 41-72. Springer, Cham 2019.

Zadebagheri, M., Azarpanah, A., Javanmardi, S.: Proline metabolite transport an efficient approach in corn yield improvement as response to drought conditions. - Int. J. Farm. Allient. Sci. 3: 453-461, 2014. 